\title{
Solid State Differentiation of Plasma Thiols using a Centrifugally Activated Mercaptobenzothiazole Disulphide Exchange Indicator
}

\author{
Samir Chahine, ${ }^{a}$ Callum Livingstone ${ }^{b}$ and James Davis ${ }^{* a}$ \\ Receipt/Acceptance Data [DO NOT ALTER/DELETE THIS TEXT] \\ ${ }_{5}$ Publication data [DO NOT ALTER/DELETE THIS TEXT] \\ DOI: 10.1039/b000000x [DO NOT ALTER/DELETE THIS TEXT]
}

\begin{abstract}
The solid state interaction of mono and macromolecular thiols at a disulphide heterocycle is shown to provide a versatile pathway for their speciation.
\end{abstract}

10 The concentration of anti-oxidant thiol (RSH) within plasma has been shown to be a potentially valuable semi-quantitative indicator through which the severity of various injuries or diseases can be assessed $^{1-6}$. While Ellmans Reagent (5,5'-dithio-(2-nitrobenzoic acid) $)^{7}$ has long been the mainstay of routine thiol analysis, it is 15 unable to speciate between sulphydryl albumin and the low, monomolecular species such as glutathione (GSH), cysteine (CSH) and homocysteine ( $\mathrm{HCSH})$ and, as a consequence, can only deliver total plasma thiol (PSH) concentration. The latter is widely recognized as having clinical merit ${ }^{4}$ but there is considerable debate 20 as to whether mono, macro or total thiol respond in equal measure to the onset of physiological stress ${ }^{4-6,8-10}$. The ability to differentiate between the different components invariably requires more elaborate liquid chromatographic analysis and hence requires the referral of the sample to specialist laboratories with the time delays 25 often negating the diagnostic advantage. The present communication presents a wholly new but integrated strategy for plasma thiol analysis that provides the clinician with ready access to a procedure that could distinguish between these groups. This could thereby offer the possibility of facilitating a better 30 understanding of the PSH/RSH metabolomics.

The basic strategy is outlined in figure 1 and is highlighted by the passage of cysteine (I) through a centrifugal filter packed with a 2,2'-dithio(bis)benzothiazole (II) indicator. The latter is insoluble within aqueous solution and serves as a densely packed particulate 35 filter. In principle, the disulphide should react with free sulphydryl thiols through a mechanism analogous to that observed with Ellman's Reagent (ER) resulting in the formation of the mixed disulphide (III) and the release of the mercaptobenzothiazole anion (IV).

40 Spectroscopic investigation (uv/vis and $1 \mathrm{H}$ NMR) of the filtrate revealed that the principal product in the filtrate was in fact the mixed disulphide(III) and not the free mercaptobenzothiazole (IV). It appears that the latter, like its disulphide parent, is insoluble within the buffer and is retained within the packing material. The 45 addition of the amino acid functionality to the complementary

${ }^{a}$ Chemistry, School of Biomedical and Natural Sciences,Nottingham Trent University, Nottingham, NG11 8NS, Tel: +44 (0) 115848 3218, E-mail: james.davis@ntu.ac.uk

${ }^{b}$ Royal Surrey County Hospital NHS Trust, Guildford, GU2 7XX, UKAddress, Address, Town, Country. Fax: $X X X X X X X X X X$; Tel: $X X$ XXXX XXXX; E-mail: xxxx@aaa.bbb.ccc

$†$ Electronic Supplementary Information (ESI) available: [product characterisation and experimental details]. See http://dx.doi.org/10.1039/b000000x/

This journal is (c) The Royal Society of Chemistry [year] thiazole component serves to aid solubilisation and, as such, is preferentially released. Similar results were obtained for homocysteine and glutathione.

50

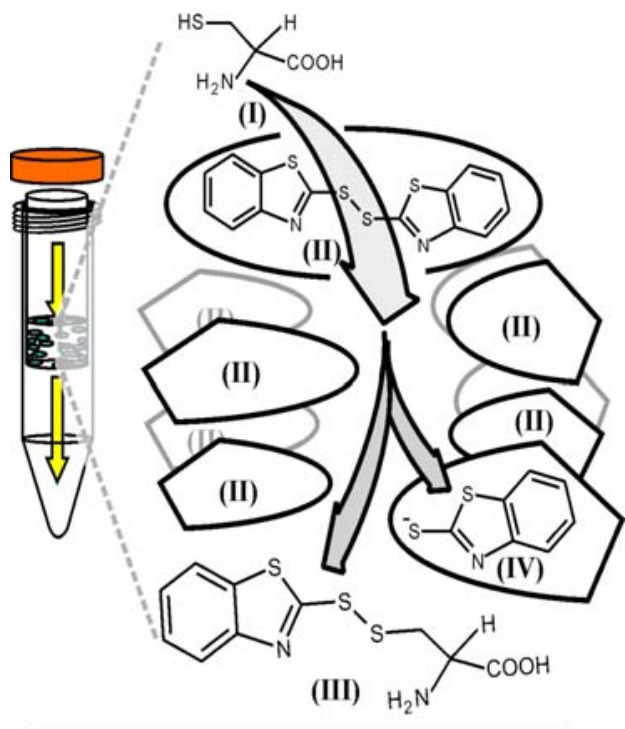

Fig 1. Centrifugal Filter Device (CFD)

An assessment of the percentage conversion of the free thiol into the mercaptobenzothiazole (MBT) conjugate was conducted (employing a before and after spectroscopic methodology) with the recovery of the thiol constituents being in order of $100+/-2 \%$ 75 (based on $20 \mu \mathrm{M}$ cysteine). The uv spectroscopic profiles for additions of CSH $(40 \mu \mathrm{M})$, GSH $(40 \mu \mathrm{M})$ and bovine albumin $(0.82 \mathrm{mg} / \mathrm{L})$ are shown in figure 2 . The principal absorption bands for $\mathrm{CSH}$, GSH and HCSH moieties were observed at 312nm and correspond predominately to the MBT component of the 80 conjugates. Significantly, the passage of albumin did not lead to any change in the absorption profile from that of the corresponding unfiltered control.

The molar absorptivities (CSH, $\lambda \max =312 \mathrm{~nm}, \varepsilon=275 \mathrm{~mol}^{-1} \mathrm{~L}$ $\mathrm{cm}^{-1}$ and GSH, $\varepsilon=365 \mathrm{~mol}^{-1} \mathrm{~L} \mathrm{~cm}^{-1}$ ) are significantly less than that 85 of ER $\left(\lambda \max =412 \mathrm{~nm}, \varepsilon=14150 \mathrm{~mol}^{-1} \mathrm{~L} \mathrm{~cm}^{-1}\right)$ and can be attributed to the lack of the nitrothiolate chromophore. It can be inferred that the added complexity of the proposed procedure and poor sensitivity would fail to make this a realistic competitor to existing spectroscopic protocols. However, the prime advantage 90 lies in the interaction of the thiol component with the particulate packing. As 2,2'-dithio(bis)benzothiazole is essentially insoluble, the exchange is dependent upon the target reacting directly with the solid packing as it is forced through the filter. The key question in 
the present investigation revolved around whether sterically buried 95 thiol functionalities, ie those within albumin, were capable of interacting with the solid. While such groups are accessible to freely diffusible derivatisation agents such as ER, it could be anticipated that the protein shield would prevent the intimate and specific contact required for reaction in the present case. The assay 100 could therefore allow a specificity, as yet, unattainable using conventional wet chemical techniques.

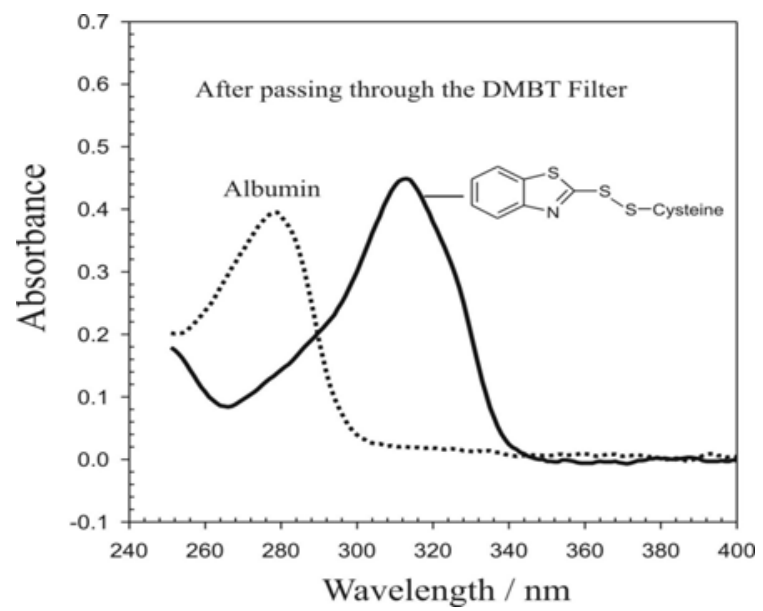

105 Fig 2. Spectroscopic profiles of CFD processed MBT conjugates compared with that of an albumin sample.

The absence of the MBT spectroscopic profile from the CFD processed albumin, figure 2, would suggest that the protein did not 110 react. This preliminary result was followed up by passing a series of albumin solutions ( 0.02 to $0.82 \mathrm{mg} / \mathrm{L})$ through the modified filter with the filtrates analysed for thiol through the addition of ER. In contrast to the previous experiments with the monomolecular thiols, positive results were obtained. The recovery of ER active 115 albumin was typically $100+/-1 \%$ (RSD $=1.5 \%, \mathrm{~N}=3$ based on $0.82 \mathrm{mg} / \mathrm{L} \mathrm{AlbSH}$ ) and serves to confirm our supposition that direct and specific interaction at the solid interface is required for disulphide exchange to occur.

The clinical efficacy of the system for discriminating between 120 albumin, low molecular weight thiols and indeed other physiological components was then assessed through examining the plasma thiol profiles of three individuals (2M, 1F). Plasma samples (500 uL) were passed directly through the filter without any form of pretreatment. The filtrate was first analysed using 125 direct uv spectroscopic analysis as indicated previously (figure 2).

The absence of any appreciable absorbance within the plasma at $312 \mathrm{~nm}$ should therefore allow any increase at this wavelength, subsequent to CFD processing, to be attributed predominantly to the presence of MBT conjugates. The magnitude of the latter 130 providing an immediate estimation of low molecular weight thiol concentration. However, there is an inherent limitation to this approach in that the spectroscopic properties of the conjugate will differ depending on the nature of the amino acid substituent. This is evident from the molar absorptivity data presented previously for 135 the GSH, CSH and HCSH variants. The data obtained is presented in table 1 and was analysed using the regression data from both
GSH and CYS calibration runs, the difference between the two estimates in practice have a negligible bearing on the measurement with the differing determinations of total low molecular weight 140 thiols being well within the experimental error for either process.

\begin{tabular}{lccc}
\hline $\begin{array}{l}\text { Direct CFD } \\
\text { Analysis }\end{array}$ & M1 & M2 & F1 \\
\hline & & & \\
$\mathrm{RSH}_{(\mathrm{GSH})} / \mathrm{mM}$ & 0.147 & 0.164 & 0.186 \\
$\mathrm{RSH}_{(\mathrm{CSH})} / \mathrm{mM}$ & 0.142 & 0.164 & 0.193 \\
\hline $\begin{array}{l}\text { Each based on 3 replicate measurements } \\
\end{array}$
\end{tabular}

Table 1. Direct uv/vis analysis of plasma RSH concentration

A series of secondary experiments were then conducted using 155 ER before and after the passage of plasma through the modified filter device. The results are detailed in table 2. Ellman analysis of the plasma before filtration yields the total plasma thiol concentration (PSH) with the subsequent filtrate providing albuminSH - given that the low molecular weight species will have 160 reacted to form the ER inactive MBT conjugates.

\begin{tabular}{lccc}
\hline CFD/ER & & Subject & \\
Analysis & M1 & M2 & F1 \\
\hline
\end{tabular}

\section{(A) ER Before CFD}

Total PSH / mM $\quad 0.530 \quad 0.778 \quad 0.650$

\section{(B) ER After CFD}

\begin{tabular}{lccc} 
AlbSH $/ \mathrm{mM}$ & 0.391 & 0.615 & 0.457 \\
$\mathrm{RSH}_{(\mathrm{A}-\mathrm{B})} / \mathrm{mM}$ & 0.139 & 0.163 & 0.193 \\
\hline \multicolumn{4}{l}{ Each based on 3 replicate measurements }
\end{tabular}

175 Table 2. CFD/ER uv/vis analysis of plasma RSH concentration.

It can be assumed that the subtraction of the albuminSH from the PSH result should yield the low molecular weight thiol concentration. The estimates shown in table 2 are in excellent 180 agreement with the direct uv spectroscopic determination. This again confirms that, despite the minor variation in uv/vis sensitivity between different MBT-SR conjugates, the procedure is indeed clinically robust.

It could be anticipated that chemical manipulation of the 185 disulphide to incorporate a chromophore (such as the nitro group common to ER) or an electro-responsive redox substituent would provide a significant enhancement to the basic assay performance. The use of quinoid indicators to facilitate potentiometric detection - essentially acting as an electrochemical Ellmans test - is also 190 possible with the filter packing providing the all important differentiation between macro and mono molecular thiols ${ }^{10,11}$.

The system also provides an effective means through which albumin can be assessed as this is increasingly being viewed as an important diagnostic in its own right ${ }^{8-10}$. The standard adsorptive 195 dye methodologies such as bromocresol green (BCG) assays that 
are currently used can be subject to interference ${ }^{13-16}$ and hence the approach advocated here could allow a more robust route to analysis. The clinical viability of the latter was assessed and the results obtained from the centrifugal system compared with the 200 BCG test. The preliminary results are detailed in table 3 and show a good correlation with the standard assay.

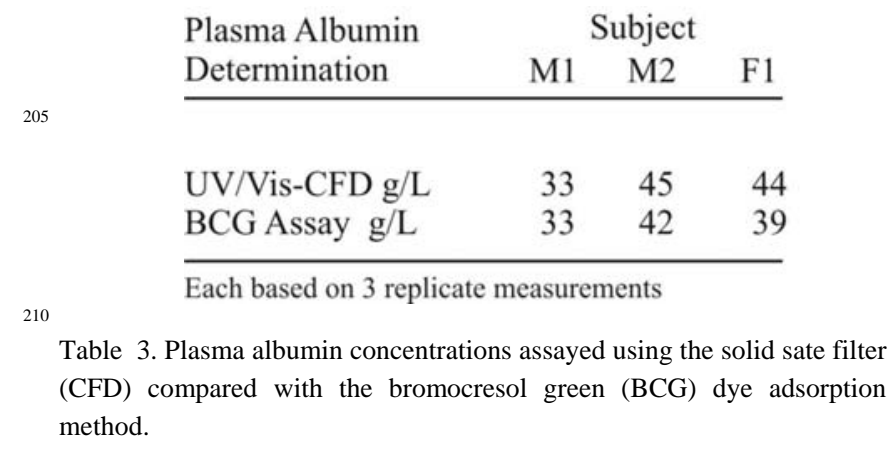

215 The system is clearly a vesratile assay that can be used to effect the speedy, accessible, and accurate speciation of physiologically significant thiol groupings. It can thus aid the differentiation between mono and macromolecular species and provides distinct routes through which both can be easily quantified. The system is 220 readily attainable and provides a simple route through which a key clinical need could be addressed. The solid state differentiation capabilities presented by the disulphide could also be exploited in a variety of other formats and is not limited solely to the centrifugal separation exploited here. It could serve as an selective coating in 225 sensors or a pre or post column derivatising step in LC applications. It is clear however that the protocol presented thus far provides a novel, robust and generic foundation from which this further work can be pursued.

${ }_{230}$ Acknowledgment. The authors thank the EPSRC for Financial support and South West Surrey LREC for granting permission to conduct the clinical trial.

\section{Notes and references}

1 I.C. West, Diabetic Medicine, 2000, 17, 171

2352 S.E. Moriarty, J.H. Shah, M. Lynn, S. Jiang, K. Openo, D.P. Jones, P. Sternberg. Free Rad Biol Med., 2003, 35, 1582

3 R.P. Robertson, J. Harmon, P.O. Tran, Y. Tanaka, H. Takahashi. Diabetes, 2003, 52, 581

4 D.J. VanderJagt, J.M.Harrison, D.M. Ratliff, L.A.Hunsaker, D.L.

240 VanderJagt, Clin. Biochem., 1999, 34, 265

5 P.J. Wlodek,O.B. Smolenski, G. Chwatko, M.B. Iciek, A. Milkowski, E. Bald, L. Wlodek. Clin Chim. Acta, 2006, 366137

6 R. Kachadourian, B.J. Day. Free Rad. Biol. Med., 2006, 4165

7 G. Ellman, Archives of Biochemistry and Biophysics, 1959, 82, 70

2458 H.O. Olawumi,P.O. Olatunji. HIV Med. 2006, 7, 351

9 Y.M. He, X.J. Yang, J. Hui, T.B. Jiang, J.P. Song, Z.H. Liu, W.P. Jiang. Acta Cardiologica, 2006, 61, 333

10 K. Kalantar-Zadeh, R.D. Kilpatrick, N. Kuwae, C.J. McAllister, H. Alcorn, J.D. Kopple, S. Greenland. Nephrology Dialysis Transplantation, 2005, 20, 1880

11 S. Gracheva, C. Livingstone, J. Davis, Anal. Chem., 2004, 76, 3833

12 L. Yonge, S. Gracheva, S.J. Wilkins, C. Livingstone, J. Davis, J. Am. Chem. Soc. 2004, 126, 7732

13 M.M. Payn, D. Lawrence, R. Willis, E.J. Lamb. Annals Clin. Biochem. 2002, 39, 311

14 J.M. McGinlay, R.B.Payne. Annals Clin. Biochem. 1988, 25,417
15 R. Calvo, R. Carlos, S. Erill. Int. J. Clin. Pharmacol. Therapeutics, 1985, 23, 76

16 A. Uldall. J. Chin. Chem. Clin. Biochem., 1984, 22, 305 
Single column figure/scheme (below)

$\mathrm{X}$

Fig./Scheme XX Caption.

Double column figure/scheme (below)

$\mathrm{X}$

Fig./Scheme XX Caption.

Single column image (no caption) (below)

$\mathrm{X}$

Double column image (no caption) (below)

$\mathrm{X}$

Single column numbered equation/reaction (below)

$\mathrm{X}$

$(\mathrm{X})$

Single column table (below)

Table XX Caption

$\mathrm{X}$

${ }^{a}$ Footnote text.

Double column table (below)

Table XX Caption

$\mathrm{X}$

${ }^{a}$ Footnote text. 